\title{
Application Status and Trend of Sensors in Domestic Engineering Field
}

\author{
Binbin $\mathrm{Xu}^{1,2,3,4^{*}}$ \\ ${ }^{1}$ Tianjin Port Engineering Institute Co. Ltd. of CCCC First Harbor Engineering Co. Ltd., Tianjin, 300222, China \\ ${ }^{2}$ CCCC First Harbor Engineering Co. Ltd., Tianjin, 300461, China \\ ${ }^{3}$ Key Lab. of Geotechnical Engineering of Tianjin, Tianjin, 300222, China \\ ${ }^{4}$ Key Lab. of Geotechnical Engineering, Ministry of Communication, Tianjin, 300222, China
}

\begin{abstract}
From the perspective of engineering field, this paper introduces the application of sensors in engineering field, and then forecasts the application trend of sensors in engineering field by introducing the status quo of sensors in engineering field, especially in the field of comprehensive treatment of water environment.
\end{abstract}

\section{Preface}

With the development and progress of society, engineering construction has played a leading role [1]. However, due to the nature of the project construction, it is difficult to get a detailed understanding of the whole construction process [2]. Moreover, the construction environment and terrain conditions of many engineering projects are complex, so it is difficult to fully consider the influence of the surrounding environment and geographical and topographical conditions on the construction, thus affecting the construction progress and increasing the cost. The traditional manual monitoring and monitoring construction method is long time, high cost, low efficiency, and information acquisition is not timely, which makes it difficult to carry out the construction monitoring and monitoring collaborative work [3]. In the whole process of engineering construction, it is particularly necessary to use sensor technology to collect the whole process data of engineering construction to guide the construction [4].

The engineering field sensor [5] is a kind of equipment that uses information technology to perceive the construction process and construction process of civil engineering. By analysing the data monitored by the sensor, it can feed back the whole construction process in a real-time way, so that the engineering personnel can understand the whole construction process in detail. It can guide the engineering personnel to find solutions to construction problems and improve the engineering quality and efficiency with saving construction costs.

\section{Present situation}

The research and application of foreign sensor technology began in the mid-20th century, and successfully applied the sensor technology to the construction monitoring in the engineering field [6]. So far, the application of foreign sensors in construction is more and more extensive, which supports the completion of monitoring in the construction process. Domestic sensor research and application development started relatively late. After recent years of development, sensor types have also had a certain scale [7]. However, the application of sensors in the same type and different types of projects are also different. There is no systematic scheme to sort out the current situation of sensor application in engineering field, so it is necessary to understand the development and application status of sensors in engineering.

At present, the types of sensors applied in engineering field mainly include electrical sensor, photoelectric semiconductor sensor, charge sensor, potential sensor, resonant sensor, electrochemical sensor and so on.

\subsection{Electrical sensor}

The electrical sensor is based on ionic conduction, which is widely used in non-electrical measurement technology. Due to the different forms of electrical characteristics, electrical sensors are also divided into resistance sensor, capacitive sensor, inductive sensor, magnetoelectric sensor and eddy current sensor.

1) Resistance sensor is made by using rheostat to convert non-electric quantity into resistance signal. Resistance sensors generally have potentiometer type, contact resistance type, resistance strain gauge and piezoresistive sensor. Resistance sensor is mainly used to measure displacement, pressure, force, strain, torque, air flow velocity, liquid level and liquid flow in engineering.

2) Capacitive sensor is made by changing the geometric size of the capacitance or changing the nature and content of the medium, so as to change the

\footnotetext{
* Corresponding author: xubinbin@tpei.com.cn
} 
capacitance. It is mainly used for the measurement of pressure, displacement, liquid level, thickness, moisture content and other parameters in engineering.

3) Inductive sensor is made by changing the magnetic circuit geometry size and magnet position to change the inductance or mutual inductance or the principle of piezomagnetic effect. It is mainly used to measure displacement, pressure, force, vibration, acceleration and other parameters.

4) Magnetoelectric sensor is made by using the principle of electromagnetic induction to convert the measured non-electric quantity into electric quantity. It is mainly used for the measurement of flow, speed, and displacement.

5) The eddy current sensor is made by using the principle that the metal moves in the magnetic field to cut the magnetic line of force and forms eddy current in the metal. It is mainly used to measure displacement and thickness.

6) The charge sensor is made by using the principle of piezoelectric effect, which is mainly used for the measurement of force and acceleration.

7) The potential sensor is made of thermoelectric effect, photoelectric effect and Hall effect. It is mainly used to measure temperature, magnetic flux, current, speed, light intensity, thermal radiation and other parameters. Electrical sensors are widely used in engineering fields. For example, Liang has achieved good results in real-time monitoring of axial force and bending angle of force measuring anchor rod by using resistance sensor [8]. Chen used capacitive sensor to monitor the moisture content of manufactured sand in real time, which provided a basis for concrete quality monitoring [9]; Liu et.al used LVDT differential inductive displacement sensor to measure well diameter. This electronic measurement method has a good application prospect in open hole oil logging tools [10].

\subsection{Photoelectric sensor}

Photoelectric sensor is made of photoelectric effect and optical principle of photoelectric devices. Photoelectric effect will be produced after being exposed to visible light, and the optical signal will be converted into electrical signal output. In addition to measuring light intensity, it can also measure a variety of physical quantities such as size, displacement, velocity and temperature by means of transmission, occlusion, reflection and interference of light. In photoelectric measurement, there is no direct contact with the measured object, and the beam quality is approximately zero. There is no friction and almost no pressure on the measured object in the measurement. Therefore, in many applications, photoelectric sensor has obvious advantages over other sensors. Its disadvantage is that in some applications, optical and electronic devices are more expensive and require higher environmental conditions. For example, Zhou and $\mathrm{Ou}$ of Harbin Institute of technology analysed the temperature sensing characteristics of fibres Bragg grating and the principle and method of temperature compensation for strain sensing, developed a kind of fibres brag grating packaged sensor for engineering application, and established a large-scale and distributed fibres brag grating intelligent monitoring system including sensor, optical switch, data acquisition and control software It has been successfully applied to the actual construction of the bridge. The temperature sensor and building materials are combined to monitor the local health of the bridge, and good results have been achieved [11]; Zhang et al. Analysed the characteristics of the distributed optical fibres vibration sensor and successfully applied it to the system identification of submarine photoelectric composite cable monitoring [12].

\subsection{Semiconductor sensor}

Semiconductor sensor is made of piezoresistive effect, internal photoelectric effect, magnetoelectric effect and material change caused by contact between semiconductor and gas. It is mainly used to measure temperature, humidity, pressure, acceleration, magnetic field and harmful gas. For example, Wang et al. Designed a graphene pressure sensor based on the characteristics of graphene, analyzed the relationship between the pressure of the sensor and the center deformation displacement, and combined with the static nonlinear analysis unit of ANSYS software, Numerical analysis and finite element simulation were carried out for the deflection deformation characteristics of the graphene film, and it was successfully applied to the engineering pressure monitoring and achieved good results [13].

\subsection{Resonant sensor}

The resonant sensor is made by changing the inherent parameters of electricity or machinery to change the resonant frequency, and the measured parameters are converted into frequency signals by using resonance elements, which is also called frequency sensor. It has the advantages of small size, light weight, compact structure, high resolution, high precision and convenient data transmission, processing, and storage. According to different resonant elements, resonant sensors can be divided into vibrating wire type, vibrating cylinder type, vibrating beam type, diaphragm type and piezoelectric resonance type (see vibrating wire sensor, vibrating cylinder sensor, vibrating beam sensor, diaphragm sensor, quartz crystal resonant sensor). Resonant sensors are mainly used to measure pressure, torque, density, acceleration and temperature.

\subsection{Electrochemical sensor}

Electrochemical sensors are based on ionic conductivity. According to the formation of their electrical characteristics, electrochemical sensors can be divided into potential sensors, conductivity sensors, electrical sensors, polarographic sensors and electrolytic sensors. Electrochemical sensors are mainly used to analyse gas, 
liquid or solid components dissolved in liquid, $\mathrm{pH}$ value of liquid, conductivity, and redox potential measurement.

\section{Water environment sensor}

With the development of domestic water environment comprehensive treatment, the development of water supply environment monitoring has brought great opportunities. Water environment monitoring refers to the use of chemical methods, physical methods, and biological methods to monitor the water environment.

There are two kinds of chemical methods: chemical analysis method and instrumental analysis method. Chemical analysis method is based on the chemical characteristics of substances and is suitable for constant analysis. The equipment is simple, and the accuracy is high, but the operation is relatively time-consuming. Physical methods (such as remote sensing technology) can only make qualitative description and must be combined with chemical methods to describe the situation of water body quickly and widely.

Biological method is based on the principle that organisms adapt to the environment, and indirectly judge the water quality by measuring the changes of aquatic organisms. The commonly used methods are: 1 . Indicator organisms refer to organisms that live in a certain water environment and show sensitive symptoms or even die out when the quality of water environment changes. Observing and measuring the changes of individual and population of indicator organisms can accurately judge the environmental quality status. 2 . Community structure method. Community structure refers to the composition of certain species of animals, plants and microorganisms that exist in a certain range (or region) of nature. The water quality can be judged by monitoring the changes of the community structure of aquatic organisms. 3. Bioassay. That is to use the symptoms of physiological function changes after aquatic organisms are poisoned by pollutants to judge the water pollution status. 4. Determination of residual toxicity. Organisms absorb all kinds of pollutants from the environment, migrate, transform and redistribute in the body, and accumulate in the form of residual poison. The residual toxic content in organisms is often many times higher than that in the surrounding environment. Determination of residual poison content in organism can judge the status of water pollution.

At present, water quality sensors, ORP sensors, $\mathrm{pH}$ sensors, COD sensors, BOD sensors, residual chlorine sensors, TOC sensors, conductivity sensors, turbidity sensors, dissolved oxygen sensors and so on.

1) water quality sensor, also called ammonia nitrogen sensor, is made of spray cleaner by the three-electrode system. Ammonium is the main method of measurement. Three advantages of ammonia nitrogen sensor: a. Fast and accurate measurement of ammonium ion, easy to maintain. b. Clean the sensor with pressurized water or air. c. The amplified signal allows up to 200 meters between the sensor and the analyser.

2) ORP sensor, which combines with the existing CTD detection system, develops an integrated shallow water sensor and integrated electronic equipment. The sensor consists of a pressure balanced platinum electrode and a reference electrode $(\mathrm{Ag} / \mathrm{AgCl})$ on a plastic rod. It is equipped with a ceramic diaphragm with a large number of pores. The electrolyte is a $\mathrm{KCl}$ which contains silver free gel and can be measured in $\mathrm{H} 2 \mathrm{~S}$ and sulfide.

3) $\mathrm{PH}$ sensor is composed of sensor and secondary meter. It can be equipped with three or two composite electrodes to meet the needs of various applications. Equipped with pure water and ultrapure water electrodes, it can be used for $\mathrm{pH}$ measurement of water quality with conductivity less than $3 \mu \mathrm{s} / \mathrm{cm}$ (such as chemical makeup water, saturated steam, condensate water, etc.) s290c series $\mathrm{pH}$ sensors are used.

4) Cod sensor, namely chemical oxygen demand sensor, refers to the amount of oxidant consumed by oxidizing reducing substances in 1 liter water sample under certain conditions, which is converted into $\mathrm{mg} / \mathrm{L}$ of oxygen required after each liter of water sample is oxidized. It reflects the degree of water pollution by reducing substances. This index is also used as one of the comprehensive indexes of relative content of organic matter.

5) BOD: biochemical oxygen demand or biochemical oxygen demand (five-day chemical oxygen demand), which represents a comprehensive indicator of the content of organic matters and other aerobic pollutants in water. It shows that the total amount of dissolved oxygen in water is consumed when organic matter in water is oxidized and decomposed due to the biochemical action of microorganism, which makes it inorganic or gasified.

6) Residual chlorine sensor is a kind of galvanometer sensor with two electrodes coated with thin film. It is used to detect the residual chlorine concentration in water without surfactant. It can also be used in seawater. Its typical applications include swimming pool water treatment, drinking water disinfection or similar water quality treatment.

7) TOC sensor, also known as total organic carbon (TOC), is an important indicator to analyze organic pollution in water samples, and TOC sensor is often used in water quality analysis of pharmaceutical industry.

8) Conductivity sensor is used in laboratory, industrial production and detection fields to measure the conductivity of ultrapure water, pure water, drinking water, sewage and other solutions or the concentration of the whole water sample ions.

9) Turbidity sensor is a kind of low-cost sensor specially used in household appliances. It is mainly used to measure the water turbidity of washing machines, dishwashers and other products. The best washing time can be determined by measuring the degree of water pollution to judge the cleanliness of the washed articles.

10) Dissolved oxygen sensor is a kind of sensor used to measure the dissolved amount of oxygen in water.

In the past 20 years, the water quality of Lingyang River Estuary and the Pearl River Delta has been monitored simultaneously with the water quality monitoring of the Pearl River Delta and the Pearl River Delta. Based on the characteristics of biological community structure, biodiversity index and water 
quality indicator organisms, this paper summarizes the biological monitoring methods suitable for the Pearl River Delta and evaluates the water pollution degree. Reasonable use of various new analytical instruments and technologies has formed the ability of monitoring and analyzing aquatic animals, aquatic plants, benthos, periphytes and residual toxicity of organisms related to water environment and accumulated a lot of experience and valuable data.

At the same time, it is also concerned that many domestic scholars have made a lot of attempts to use biological methods to monitor the water environment. For example, in Lanzhou section of the Yellow River, the population distribution and variation of benthos and the relationship with their related chemical indexes and hydrological characteristics are summarized, and the growth and decline laws of aquatic organisms are summarized, and the biological evaluation of water quality in Lanzhou section of the Yellow River is carried out; Suzhou River: the relationship between ecological changes of periphyton community structure and water quality; Huangpu River: the application of periphyton community in water quality monitoring Zhao Huixin, Li $\mathrm{Yi}$ et al. Developed an electrochemical sensor for monitoring the concentration of trace heavy metals in water environment by using mercury film glassy carbon disc electrode. Based on the differential pulse anodic stripping voltametric method, the simultaneous determination of zinc, cadmium, lead and copper has been realized, which has a good effect in the application of water environment [15].

\section{Development trend}

At present, the application of sensor monitoring technology to the engineering field presents a certain trend. From the development status of sensor and its application in the engineering field, the research and development of sensor is moving towards the direction of miniaturization, intelligence, multi-function, systematization and durability. In particular, the application of new sensors based on photoelectric technology and biological principle technology in the engineering field has brought the innovation of monitoring technology in the engineering field.

\section{1) Miniaturization}

Sensor miniaturization refers to a kind of sensor with small volume and light weight, which adopts precision machining, and also provides microelectronics, MEMS, nano technology to realize the traditional sense of sensor reduction. In the modern engineering construction, the traditional sensor is more and more limited in volume, weight and application range. With the development of micro sensor technology, and the volume, weight and technology have great advantages, the application proportion of micro sensor in engineering construction is more and more. For example, in the field of UAV surveying and mapping, micro sensors control the flight attitude of UAV to make the drawing area deviation within a reasonable range.

2) Intellectualization
Intelligent sensor means that on the basis of meeting the basic functions of the sensor, the sensor is equipped with a microprocessor, which has the functions of collecting, processing, exchanging information, correcting deviation automatically, calibrating automatically, filtering automatically and so on. At the same time, it should also have the function of simple logic judgment and information automatic processing, and be able to correct and process the wrong signal in real time. The rise of smart site has put forward new requirements for traditional sensors. The traditional sensors have gradually failed to meet the requirements of smart sites. The sensor manufacturing industry is gradually transiting from traditional to intelligent. At the same time, the construction in the engineering field is more or less accompanied by the problem of difficult data storage, such as construction in the marine environment, tunnel engineering, etc., which requires the sensor to be able to analyze, process and store the collected data.

3) Multi-function

Sensor multifunction means that the sensor can measure two or more kinds of measurement. Due to the special influence of construction site and construction environment and the consideration of saving engineering cost, it is a waste of space and difficult to maintain multiple sensors at the same monitoring point. It is necessary for a sensor to have multiple functions. For example, a water depth sensor can measure both water depth and water pressure.

4) Systematization

Systematic sensor means that the sensor is highly integrated with microprocessor, data storage, data automatic filtering and network. That is to say, a sensor has all the functions of processing and collecting data and transmitting the data to the designated server. The server only needs to analyze and process the collected data. At present, the sensors in the engineering field are not limited to the sensor itself, but a whole set of systems including sensor, network transmission module, micro processing module, etc. in view of the relatively tight construction period of most construction projects, the installation, debugging and maintenance of traditional sensors need a certain time, which affects the progress of engineering construction. The systematic sensor only needs to install the sensor When it is placed in the designated position, networking, debugging and data acquisition are automatically completed, which no longer affects the normal construction.

5) Networking

With the development of $5 \mathrm{~g}$ and Internet of things technology, sensors as one end of the Internet of things have been easy to realize. At the same time, the construction environment in the engineering field is relatively special, and the construction environment is mostly in the areas lacking of network coverage or poor network signal. The traditional $4 \mathrm{G}$ and LAN technology brings inconvenience to sensor data transmission. It can not maintain long-term stability, high precision and long life in harsh environment. Meanwhile, theft occurs from time to time, which can not fully meet the application requirements of sensors. The application range of 
sensors supporting NB or Lora technology has been greatly improved. In the construction environment with poor signal, the sensor uses Lora communication which is far away from the transmission signal to transmit data. In the place with good signal, $\mathrm{Nb}$ signal is used to connect the sensor data information in parallel, so that the sensor data can be transmitted to the designated target. At the same time, $\mathrm{Nb}$ and Lora technology can also quickly determine the location of sensor damage, easy to replace or timely detect the occurrence of theft. 6) Durability

The durability of sensors is not only limited to the long-term use of materials used by sensors, but also should be considered from the impact of sensor application scenarios, power consumption, robustness and stability of micro processing system. The nature of construction projects determines that sensors often have to withstand the challenge of harsh monitoring environment, and the project budget is limited, so the sensors should be reused, which is conducive to saving costs and improving construction efficiency.

\section{Conclusion}

With the increasingly strict requirements of engineering quality, it is inevitable to use sensors to monitor construction quality in the engineering field. This paper introduces the current situation of sensors in engineering field from the perspective of sensor application in engineering field and looks forward to the development trend of sensors in engineering field in the future. This paper has certain guiding significance for engineering practitioners to apply monitoring sensors.

\section{References}

1. Z.B. Wang, C. Huang and Y. Li, Engineering Research 10(3), 23-246 (2018)

2. Y.G. Chen, H.Q. Chen and C.Y. Gao, Safty and Environment Engineering 26(2), 105-110 (2019)

3. Q.R. Ren, L. Ye and X.Z. Li, Manufacturing Automation 33(12), 150-152 (2011)

4. L. Hong, X. Li and S.L. Wang, Modern Manufacturing Engineering 5, 132-138 (2019)

5. D. Wang, Instrument Technique and Sensor | Instr Techn Sensor 8, 39 (2019)

6. R.J. Wu, B.C. Zhang and C.M. Kang, Journal of Sensor Technology 5, 5-7 (2003)

7. Y.L. Zhang, Automation \& Instrumentation 27(8), $56(2012)$

8. C.X. Liang, Energy and Energy Conservation 11, 17-18 (2016)

9. M. Chen, Doctor Thesis, 2020

10. J. Liu, D.H. Li and J.H. Gao, China Oil S2, 109 (2016)

11. Z. Zhou and J.P. Ou, Journal of Harbin Institution of Technology 5 (2004)
12. Y. Zhang, Modern Electronics Technique 43(22) 30-35 (2020)

13. D. Wang, Y.F. Qin and R.B. Yuan, Semiconductor Optoelectronics Semicond Opt 41(05), 676-680 (2020)

14. R. Dai H.T. $\mathrm{Yu}$ and Q. Wang, Journal of Mechanical Engineering 56(13), 50-59 (2020)

15. H.X. Zhao, Y. Li and W. Cai, Instrument Technique and Sensor | Instr Techn Sensor S1, 158-161 (2009) 